\title{
Erratum to: surface alterations of zirconia and titanium substrates after Er, Cr:YSGG irradiation
}

\author{
Persio Vasconcelos Miranda • José Augusto Rodrigues • \\ Alberto Blay • Jamil Awad Shibli • Alessandra Cassoni
}

Published online: 30 April 2014

(C) Springer-Verlag London 2014

\section{Erratum to: Lasers Med Sci DOI 10.1007/s10103-013-1516-x}

The original version of this manuscript, unfortunately, did not contain the Materials and Methods section. The said missing section is given below.

\section{Materials and methods}

Experimental design

The experimental units consisted of 20 disks ( $n=5$ per group). Ten disks of yttrium-stabilized tetragonal zirconia poly crystal (Y-TZP) (AS Technology Titanium FIX, São José dos Campos, Brazil) with $5 \mathrm{~mm}$ diameter and $3 \mathrm{~mm}$ high were standardized from CAD-CAM blocks. Ten disks with $5 \mathrm{~mm}$ diameter and $4 \mathrm{~mm}$ thickness of titanium sandblasted acid-

The online version of the original article can be found at http:// dx.doi.org/10.1007/s10103-013-1516-x.

P. V. Miranda • J. A. Shibli

Department of Periodontology and Oral Implantology, Dental

Research Division, University of Guarulhos, Guarulhos, SP, Brazil

J. A. Rodrigues $\cdot$ A. Cassoni

Department of Restorative Dentistry, School of Dentistry, Guarulhos

University, São Paulo, Brazil

A. Blay

Nuclear and Energy Research Institute (IPEN), University of São

Paulo, São Paulo, SP, Brazil

A. Cassoni $(\bowtie)$

Pós Graduação em Odontologia, Universidade Guarulhos, Praça

Teresa Cristina, 229, Centro, Guarulhos, SP, Brazil 07023-070

e-mail: acassoni@prof.ung.br

etched surface (AS Technology Titanium FIX) were used. The disks with sandblasted acid-etched surface were blasted with $100 \mu \mathrm{m} \mathrm{Al}{ }_{3} \mathrm{O}_{2}$ particles. After the sandblast, the specimens were ultrasonically cleaned with an alkaline solution, washed in distilled water and pickled with $\mathrm{HNO}_{3}$. This industrial process creates the surface referred to as sandblasted acid-etched (SLA).

Specimen treatment and laser system

The Y-TZP zirconia disks were randomized into two groups of laser irradiation: Y-TZP_G1 = control (no laser treatment); YTZP_G2 = Y-TZP irradiated with Er, Cr:YSGG laser (1.5 W; air-water cooling proportion $80 / 25 \%$ ). The titanium SLA disks were randomized into two groups of laser irradiation: TI_G1 = Titanium SLA control (no laser treatment); TI_G2 = titanium SLA irradiated with Er, Cr:YSGG laser $(1.5 \overline{\mathrm{W}}$; airwater cooling proportion $80 / 25 \%$ ). The control groups thus had surfaces left 'as is' after their production by CAD-CAM or SLA treatment.

The control groups did not receive any laser treatment (YTZP_G1 and TI_G1). Er, Cr:YSGG laser $(\lambda=2780 \mathrm{~nm}$; Water lase, Bio lase Technologies Inc, Irvine, CA, USA) was used on each Y-TZP zirconia disk (Y-TZP_G2) and titanium disk (TI_G2) in focused mode with a G4 tip (ø $378 \mu \mathrm{m})$ positioned at $1 \mathrm{~mm}\left(90^{\circ}\right)$ from the disk surface (focused mode). An endodontic $\mathrm{K}$-file fixed to the laser hand piece ensured the distance standardization. Repetition rate was fixed on $20 \mathrm{~Hz}$. The air-water cooling proportion was fixed on $80 / 25 \%$ to YTZP_G2 and TI_G2. One single trained operator uniformly irradiated by hand the disk surface in a grid pattern for $30 \mathrm{~s}$. The hand pice was positioned perpendicularly to the disk surface. Each sample was irradiated once in each direction, moving the hand piece slowly horizontally and vertically, to promote homogeneous irradiation and cover the entire sample 
area. The energy density used for the laser irradiation of each laser group was $67 \mathrm{~J} / \mathrm{cm}^{2}$.

\section{Confocal white light microscope}

The surface topography of the disks was investigated using confocal microscope (Leica Scan DCM 3D, Leica Microsystems Ltd, Switzerland) with objective magnification $50 \times$, numeral aperture 0.9 , optical resolution $(\mathrm{X} / \mathrm{Y}) 0.16 \mu \mathrm{m}, \mathrm{FOV}(\mu \mathrm{m})$ is $254.64 \times 190.90$. The vertical resolution was $<3 \mathrm{~nm}$ with sub-micron lateral resolution in confocal mode according to manufacturer. The control groups (TI_G1 and Y-TZP_G1) were used as references. Profiler software (Leica DCM 3D Dual Core, Leica Microsystems Ltd) calculated the surface roughness. Sa and Ra were recorded. Sa measure was performed at $254.64 \times 190.90 \mu^{2}$ and Ra length of $254.64 \mu \mathrm{m}(768 \times 576$ pixels $)$.

\section{Statistical analysis}

The factor under study was laser: no laser treatment and laser treatment. Values were statistically analyzed by $t$-test at the $95 \%$ confidence level $(\alpha=0.05)$ using a software package (SANEST, EPAMIG, MG, Brazil). Power was calculated using the G power 3.1.2 software package (Heine, Universität Dusseldorf, Germany) $(p=0.93)$. The mean of $\mathrm{Sa}$ and Ra roughness values and standard deviation were calculated from five profiles for each group. 